\title{
Application of Lagrange Multiplier Formalism to Modeling of Longitudinal Vibration of Lower-Limb Exoskeleton
}

\author{
M. Skotniczny*, D. Cekus and P. Kwiatoń \\ Częstochowa University of Technology, 42-201 Częstochowa, Poland
}

Doi: 10.12693/APhysPolA.139.535

*e-mail: marcin.skotniczny@pcz.pl

\begin{abstract}
The paper formulates and solves the problem of free longitudinal vibration of the lower-limb exoskeleton using the method of Lagrange multipliers. Parts of the exoskeleton were replaced with continuous and discrete elements. The physical model includes properties characterizing the interaction between exoskeleton components, contact with the ground and the method of applying the load. On the basis of the presented model, an algorithm and a computational program were developed. It allowed to conduct the studies of the influence of selected model parameters on the longitudinal vibrations of the considered system.
\end{abstract}

topics: discrete-continuous system, exoskeleton, free vibration, Lagrange multiplier formalism

\section{Introduction}

Exoskeletons as mobile devices are exposed to diverse and varying environmental conditions. Depending on the purpose and characteristics of the user, they focus on supporting specific parts of the body [1]. When carrying various types of loads or assisting the user's movement, they must ensure reliability and safety at work [2]. Therefore, mechanisms of this type should be examined with particular care, taking into account the influence of many parameters on their reliability.

The exoskeleton, as a walking machine, is exposed to a number of overloads, e.g., associated with bump during the contact of the mechanism with the ground, which may cause a sudden increase in stresses [3]. Other undesirable phenomena are construction vibrations, which are particularly dangerous in the case of walking machines [4, 5]. They can cause problems with movement control and, in extreme cases, lead to the destruction of the mechanism [6]. Therefore, in this study, a discretecontinuous model of longitudinal vibrations of the exoskeleton of the lower limb was developed. The model takes into account the transferred mass and the influence of the ground parameters on structure vibrations. Exemplary calculations were carried out for two positions (which occur during the working cycle) of the lower limb and for variable parameters describing the ground [7].

\section{Model description}

The analyzed discrete-continuous model of the exoskeleton's lower limb and its mechanical implementation are shown in Fig. 1. The discrete-continuous model consists of two rods of length $L_{1}$ and $L_{2}$ responsible for transferring the load of the entire mechanism to the ground. The system is loaded with a mass $m$ that replaces the top of the exoskeleton components, such as structural components, batteries, controllers of drives, and the on-board computer, and takes into account additional elements such as the carried load. The movement of the mass is restrained to operate only vertically. The influence of the ground on the mechanism was modeled using a translational spring with the stiffness $K_{\mathrm{s}}$. During the duty cycle, the values of the angles $\alpha$ and $\beta$ are changed. These angles are directly bound up with the angular displacement in the joints and are included in the control system of the device.

\section{Problem solution}

The Lagrange multipliers method was used to determine the frequency of longitudinal vibrations of the analyzed model. Using the derivations from [8], the total kinetic $(T)$ and potential $(V)$ energy for the presented system can be written as:

$$
\begin{aligned}
& T=\frac{1}{2} \sum_{i=0}^{N_{1}} M_{1 i} \dot{\xi}_{1 i}^{2}+\frac{1}{2} \sum_{i=0}^{N_{2}} M_{2 i} \dot{\xi}_{2 i}^{2}+\frac{1}{2} m \dot{z}_{1}^{2}, \\
& V=\frac{1}{2} \sum_{i=0}^{N_{1}} K_{1 i} \xi_{1 i}^{2}+\frac{1}{2} \sum_{i=0}^{N_{2}} K_{2 i} \xi_{21}^{2}+\frac{1}{2} K_{\mathrm{s}} z_{2}^{2},
\end{aligned}
$$

where

$$
M_{p_{i}}=\rho_{p} A_{p} L_{p} m_{i}, \quad K_{p_{i}}=E_{p} I_{p} k_{i} / L_{p}^{3}
$$

for $p=1,2$. The values of $m_{i}$ and $k_{i}$ were also determined on the basis of the relationship taken from [8]. 
(a)

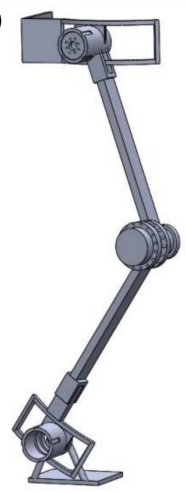

(b)

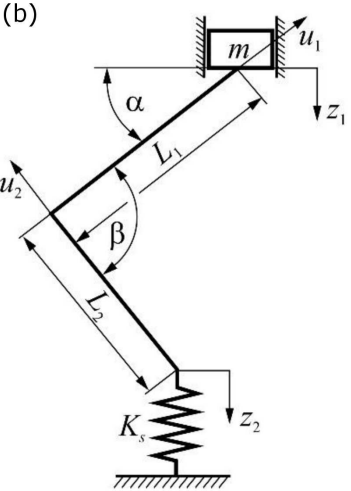

Fig. 1. The exoskeleton's lower limb: (a) 3D model, (b) discrete-continuous model.

The constraints functions, which result from the method of the joint discrete elements in the whole system as well as from the connection between the bars, are as follows:

$$
\begin{aligned}
& f_{1} \equiv u_{1}(0, t) \sin (\alpha)-z_{1}(t)=0 \\
& f_{2} \equiv u_{1}\left(L_{1}, t\right) \sin (\alpha)-u_{2}(0, t) \sin (\beta-\alpha)=0 \\
& f_{3} \equiv u_{2}\left(L_{2}, t\right) \sin (\beta-\alpha)-z_{2}(t)=0
\end{aligned}
$$

Lagrange's kinetic potential for the above set of equations has the form:

$$
L=T-V+\sum_{r=1}^{3} \lambda_{r} f_{r}
$$

where $\lambda_{r}$ are the Lagrange multipliers. Based on the Lagrange equations of the second kind, the following set of equations can be written for the system under study:

- for $i=0,1, \ldots, N_{1}$

$$
M_{1 i} \ddot{\xi}_{1 i}+K_{1 i} \xi_{1 i}=\sin (\alpha) \sum_{r=1}^{2} \lambda_{r} b_{1_{i r}}
$$

- for $i=0,1, \ldots, N_{2}$

$$
M_{2 i} \ddot{\xi}_{2 i}+K_{2 i} \xi_{2 i}=\sin (\beta-\alpha) \sum_{r=2}^{3} \lambda_{r} b_{2_{i r}}
$$

with conditions to fulfil

$$
m \dot{z}_{1}+\lambda_{1}=0, \text { and } \quad K_{\mathrm{s}} z_{2}+\lambda_{3}=0 .
$$

In the case of (6) and (7), the denotations are introduced:

$$
\begin{aligned}
& b_{1_{i r}}=U_{1_{i}}\left(x_{r}\right), \text { for }\left\{\begin{array}{l}
r=1 \Rightarrow x_{r}=0, \\
r=2 \Rightarrow x_{r}=L_{1},
\end{array}\right. \\
& b_{2_{i r}}= \begin{cases}-U_{2_{i}}\left(x_{r}\right) & \text { for } r=2 \Rightarrow x_{r}=0, \\
U_{2_{i}}\left(x_{r}\right) & \text { for } r=2 \Rightarrow x_{r}=L_{2} .\end{cases}
\end{aligned}
$$

To determine the frequency of free vibrations, we have to derive the set of equations for this purpose. We assume the harmonic solution of the system $(6-8)$, in the form:

$$
\begin{aligned}
& \xi_{n_{i}}=A_{n_{i}} \sin (\omega t) \text { for } n=1,2, \\
& z_{l}=Z_{l} \sin (\omega t) \text { for } l=1,2, \\
& \lambda_{r}=\Lambda_{r} \sin (\omega t) \text { for } r=1,2,3 .
\end{aligned}
$$

After inserting the dependences (10) into the set of equations (6) and (7), one obtains the amplitude values of the harmonic solution, i.e.,

$$
\begin{aligned}
& A_{1_{i}}=\frac{\sin (\alpha)}{K_{1 i}-\omega^{2} M_{1 i}} \sum_{r=1}^{2} \Lambda_{r} b_{1_{i r}}, \\
& A_{2_{i}}=\frac{\sin (\beta-\alpha)}{K_{2 i}-\omega^{2} M_{2 i}} \sum_{r=2}^{3} \Lambda_{r} b_{2_{i r}}, \\
& Z_{1}=\frac{\Lambda_{1}}{\omega^{2} m}, \quad Z_{2}=-\frac{\Lambda_{3}}{K_{\mathrm{s}}} .
\end{aligned}
$$

The system of equations (6) and (7) can now be expressed in a matrix form as

$$
\boldsymbol{C} \overline{\boldsymbol{\Lambda}}=0,
$$

where $\overline{\boldsymbol{\Lambda}}=\left[\bar{\Lambda}_{1}, \bar{\Lambda}_{2}, \bar{\Lambda}_{3}\right]^{T}$ and $\mathbf{C}$ denotes the square matrix

$$
\boldsymbol{C}=\left[\begin{array}{ccc}
C_{1_{11}} \sin ^{2}(\alpha) & C_{1_{12}} \sin ^{2}(\alpha) & 0 \\
+\varepsilon_{1} & C_{1_{22}} \sin ^{2}(\alpha) & \\
C_{1_{21}} \sin ^{2}(\alpha) & +C_{2_{22}} \sin ^{2}(\beta-\alpha) & C_{2_{23}} \sin ^{2}(\beta-\alpha) \\
& +\varepsilon_{2} & \\
0 & C_{2_{32}} \sin ^{2}(\beta-\alpha) & C_{2_{33}} \sin ^{2}(\beta-\alpha) \\
& & +\varepsilon_{3}
\end{array}\right]
$$

with the designated parameters

$$
\begin{aligned}
& \varepsilon_{1}=-1 /\left(m \omega^{2}\right), \\
& \varepsilon_{2}=0, \\
& \varepsilon_{3}=1 / K_{s},
\end{aligned}
$$

$$
C_{n_{k r}}=\sum_{i=0}^{N_{n}} \frac{b_{n_{i k}} b_{n_{i r}}}{K_{n_{i}}-\omega^{2} M_{n_{i}}} .
$$

The equation set (11) yields the eigenvalue problem

$$
\operatorname{det}(\boldsymbol{C})=0,
$$

which enables one to calculate the free vibration frequency of the system. 


\section{Results and discussion}

On the basis of the presented mathematical model, the algorithm and computer program have been worked out and numerical calculations have been carried out. The bisection method was used to the root-finding method. The sample computations have been conducted for the following parameters: $L_{1}=0.5 \mathrm{~m}, L_{2}=0.5 \mathrm{~m}$, rods with a rectangular section of $0.02 \times 0.045 \mathrm{~m}^{2}, N_{1}=10, N_{2}=10$. The influence of:

- configuration, i.e., $\alpha=90^{\circ}, \beta=180^{\circ}$ and $\alpha=45^{\circ}, \beta=90^{\circ}$,

- carried mass, i.e., $m=50 \mathrm{~kg}, m=60 \mathrm{~kg}, m=$ $70 \mathrm{~kg}$,

- rods material (AISI 1020: $E=2 \times 10^{11} \mathrm{~Pa}$, $\rho=7700 \mathrm{~kg} / \mathrm{m}^{3}, \quad 1060$ Alloy: $E=6.9 \times 10^{10} \mathrm{~Pa}, \rho=2700 \mathrm{~kg} / \mathrm{m}^{3}$,

- stiffness of ground, i.e., $K_{\mathrm{s}}=10^{8} \div 10^{15} \mathrm{~N} / \mathrm{m}$

on the values of the first three free vibration frequencies of the exoskeleton were analyzed.

The obtained results are presented in Figs. 2-4. Figure 2 includes a legend that also concerns the remaining results.

The greatest differences in the frequency values occur for the $K_{s}$ parameter values in the range $10^{8} \div 10^{11} \mathrm{~N} / \mathrm{m}$. Analyzing the data in Fig. 2, it can be concluded that:

- in the case of the basic vibration frequency, all parameters have a significant influence on its value and:

- as the mass $m$ increases, the frequency value decreases,

- for the bent leg $\left(\alpha=45^{\circ}, \beta=90^{\circ}\right)$, the frequency values are greater about $40 \%$ than for the straight leg $\left(\alpha=90^{\circ}\right.$, $\beta=180^{\circ}$ ),

- frequency values for AISI 1020 are higher about $68 \%$ than for the 1060 Alloy;

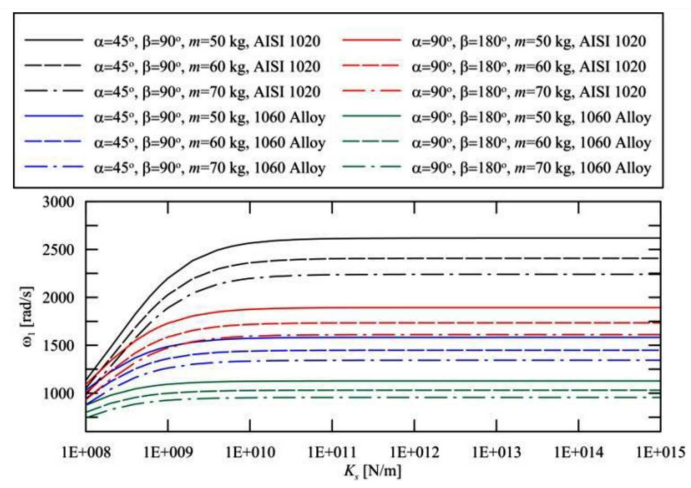

Fig. 2. First vibration frequencies of the discretecontinuous model of the exoskeleton's lower limb.

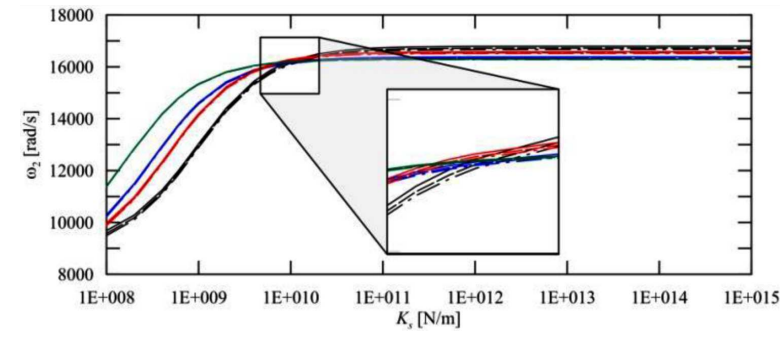

Fig. 3. Second vibration frequencies of the discretecontinuous model of the exoskeleton's lower limb.

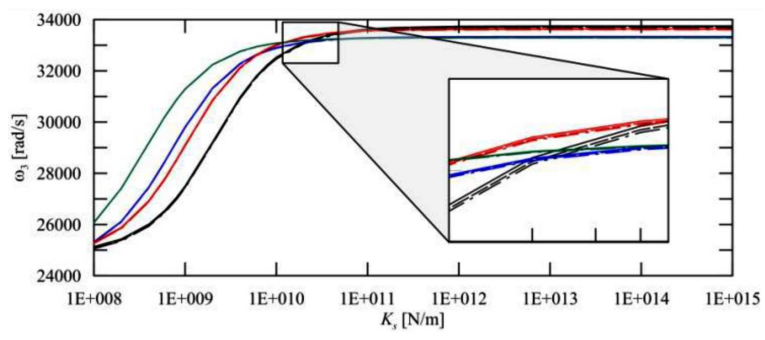

Fig. 4. Third vibration frequencies of the discretecontinuous model of the exoskeleton's lower limb.

- for other frequencies:

- the ground stiffness $\left(K_{\mathrm{s}}\right)$ in the range $10^{8} \div 10^{11} \mathrm{~N} / \mathrm{m}$ has the greatest impact,

- the effect of carried mass $m$ is negligible,

- for the $K_{\mathrm{s}}$ parameter in the range $10^{8} \div 10^{10} \mathrm{~N} / \mathrm{m}$, the frequency values for Alloy 1060 are higher (max. 15\%) than for AISI 1020, and for $K_{\mathrm{s}}$ bigger than $10^{10} \mathrm{~N} / \mathrm{m}$, the frequency values for alloy 1060 are lower $(1 \%-3 \%)$ than for AISI 1020,

- for the straight leg, the frequency values are higher (max. 10\%) than the frequency values for the bent leg (for the $K_{\mathrm{S}}$ parameter in the range $10^{8} \div 10^{10} \mathrm{~N} / \mathrm{m}$, upper of this value the impacts of $\alpha$ and $\beta$ are marginal).

\section{Conclusions}

The worked out model enables the analysis of free longitudinal vibrations of the lower-limb exoskeleton. The obtained results demonstrated that when the exoskeleton is designed, it is necessary to take into account the base on which it will move, because ground stiffness significantly affects the frequencies of longitudinal vibrations. In addition, when selecting drive systems, motors should be selected so that the frequencies produced by them do not cause resonance of the entire structure (for a given structure, they do not generate vibrations in the range from $120 \mathrm{~Hz}$ to $420 \mathrm{~Hz}$, which corresponds to the basic vibration frequency of the system depending on the operating parameters). The next stage of research will be to develop a model of transverse vibrations [9] of the discussed mechanism. 


\section{References}

[1] J.K. Proud, D.T.H. Lai, K.L. Mudie, "Exoskeleton Application to Military Manual Handling Tasks", Human Factors (2020).

[2] D. Pan, F. Gao, Y. Miao, R. Cao, T. Liu, Mech. Based Des. Struct. Mach. 43, 383 (2015).

[3] T. Wang, C. Chevallereau, C.F. Rengifo, Robot. Auton. Syst. 60, 962 (2012).

[4] T.F.C. Berninger, F. Sygulla, S. Fuderer, D.J. Rixen, in: IEEE Int. Conf. on Robotics and Automation (ICRA), Paris, 2020, p. 8726 .
[5] H. Xu, X. Zhang, X. Mu, in: 16th Int. Conf. on Ubiquitous Robots (UR), Jeju (South Korea), 2019, p. 49.

[6] S. Kajita, F. Asano, M. Moriswa, K. Miura, K. Kaneko, F. Kanehiro, in: IEEE Int. Conf. on Robotics and Automation, Karlsruhe (Germany), 2013, p. 1637.

[7] A.M. Dollar, H. Herr, IEEE Trans. Robot. 24, 144 (2008).

[8] B. Posiadała, J. Sound Vib. 176, 563 (1994).

[9] D. Cekus, Scientific Research of the Institute of Mathematics and Computer Science 10(2), 49 (2011). 